\title{
INTERCAMBIO DE EXPERIENCIAS PRO- DUCTIVAS SOBRE QUESOS ARTESANALES EN SAN LUIS DEL PALMAR, CORRIENTES
}

Lic. Lucía F. Falcione, Lic. José A. Valenzuela López, Od. Cynthya S. Sin, Mario M. Maidana, Téc. Alfredo J. Musigh, Dra. Olga M Vasek (*)

\section{RESUMEN}

Considerando la ancestral tradición de la manufactura quesera en la provincia de Corrientes y el acercamiento de pequeños productores zonales a la Universidad ocurrido en 2014. El Grupo BiMIA realizó, en el año 2015, una Jornada Práctica de elaboración de quesos en San Luis del Palmar, enfatizando las Buenas Prácticas de Manufactura y el uso de insumos tradicionales a fin de mantener esta usanza, que genera un producto de características particulares y puede ocasionar una mayor demanda, contribuyendo al desarrollo sustentable y sostenibilidad productiva de la región. Se presenta la experiencia y algunos resultados de importancia.

Palabras clave: queseros artesanos, quesos tradicionales, manufactura, tradición

\section{INTRODUCCIÓN}

En la Provincia de Corrientes existe un antiguo nicho tradicional en la producción de Quesos Artesanales, también llamados "caseros, criollos o de campo". Su producción, se realiza con leche cruda proveniente de vacas alimentadas con pastura natural, que cambia de estado líquido a sólido o semi-sólido mediante la coagulación para transformarse, tras la maduración, en el derivado lácteo que conocemos como queso. Sin embargo, para que este cambio de estado físico tenga lugar, es necesaria la adición de otro insumo: el agente coagulante. Generalmente, se prepara a partir de una porción de cuajar bovino limpio, salado y seco, por inmersión en leche, de manera tal que, las enzimas coagulantes presentes en el tejido del cuajar o librillo pasen, con ayuda de la sal, al suero rezumado en la leche. Este agente coagulante se utiliza, según la tradición correntina para "cortar la leche" durante 7-8 días, si se conserva en la heladera.

La elaboración y comercialización de este alimento manufacturado desde tiempos ancestrales se realiza de manera informal y constituye una actividad secundaria en la economía de la familia rural, situación que origina una sub-producción y un saber hacer subutilizados (Vasek y Falcione, 2013a).

Esta actividad se desarrolla en la mayoría de los establecimientos ganaderos como recurso económico complementario, en la 
cual la mujer desarrolla un rol protagónico. $\mathrm{Su}$ producción tiene un volumen apreciable y una demanda interesante por parte del mercado local, que podría ser aún más promisorio si se ofreciera al consumidor un producto seguro y de calidad uniforme (Vasek et al., 2008). Sin embargo, factores múltiples no permiten que estos pequeños productores familiares, desarrollen un crecimiento productivo de importancia zonal.

\section{ANTECEDENTES}

Durante los años de investigación del Grupo Biotecnología Microbiana para la Innovación Alimentaria (BiMIA), vinculados a la quesería artesanal (que datan de 1995) este equipo, evolucionó en este último tiempo hacia el ámbito socio-humanístico-productivo tras plantearse para quién venía trabajando. Pregunta que derivó en una sencilla respuesta "ayudar a los productores, para que esta actividad, secundaria en su vida laboral, se transforme en actividad principal con ganancias monetarias redituables que les permita vivir honradamente".

Con el objetivo de dar inicio al proceso de revalorización de la quesería artesanal, en 2014 se llevó a cabo una capacitación impulsada y coordinada por el Grupo.

Especialistas en la temática productiva de Derivados Lácteos, del Instituto $\mathrm{Na}^{-}$ cional de Tecnología Industrial-Rafaela (Santa Fe), el Lic. M. Bonafede e Ing. B. Aimar, dictaron un "Taller de elaboración de Quesos en pequeña escala" en la FaCENA, destinado a productores de la región. Estos especialistas, llegaron hasta Corrientes para compartir e intercambiar sus experiencias técnicas con los productores locales y a la vez nutrirse de sus conocimientos. "Desde el 2011 interactuamos con productores queseros, mediante talleres teórico-prácticos sobre elaboración artesanal para fortalecer sus valiosos conocimientos empíricos obtenidos de la experiencia y que les fueran transmitidos por generaciones anteriores" remarcó el Lic. Bonafede.

A su vez, el Ing. Aimar destacó haber encontrado trabajos de investigación realizados en la UNNE, cuya temática y avances le causaron admiración, ya que en 2009 , les resultaron "adelantados" en lo referente a elaboración de insumos para la escala artesanal. Situación que dio origen a la vinculación con el Grupo BiMIA.

Durante esta experiencia, el acercamiento de los productores a la Universidad, en particular a FaCENA, generó la conexión necesaria para la vinculación cuyo objetivo final es contribuir al desarrollo sustentable de la producción quesera en Corrientes y el reconocimiento de los Quesos Artesanales de Corrientes como patrimonio histórico, social y cultural de la Provincia.

\section{Jornada práctica de elaboración de quesos}

Con la intención de activar el proceso que se persigue desde hace tiempo buscando reunir las voluntades de gobierno, universidad y productores para que éstos últimos logren no solo jerarquizar su producción, sino también transformarla en una actividad principal redituable apropiadamente, se impulsó el acercamiento entre estos sectores diferenciales, vinculación conocida como Triángulo de Sábato, sobre la base de que el sector generador de conocimientos (la Universidad), debe transferir a la Sociedad estos conocimientos y, si es posible, que, al menos un sector productivo (aceptor de conocimientos) pueda asimilarlos y ponerlos en práctica real.

Sobre esta base y, a partir de la expe- 
riencia previa antes mencionada, el Grupo BiMIA, en forma interdisciplinaria con la Municipalidad de San Luis del Palmar, personal del INTA, la Secretaría de Agricultura Familiar de la Nación (Delegación Corrientes) y un grupo de productores tradicionales de quesos artesanos de San Luis del Palmar, Corrientes, realizó una jornada de capacitación e intercambio de experiencias productivas sobre el tema.

La Jornada, bajo el nombre de "Jornada Práctica de elaboración de quesos", se desarrolló en la Granja Santa María, propiedad del Señor Juan Carlos Esquivel, $6^{\circ}$ sección, San Luis del Palmar, con la asistencia de productores de las distintas secciones de la mencionada Localidad y la premisa de mantener la tradición en la elaboración de estos quesos, potenciando la inocuidad del producto.

Durante la Jornada, se realizó una producción de quesos destacando las Buenas

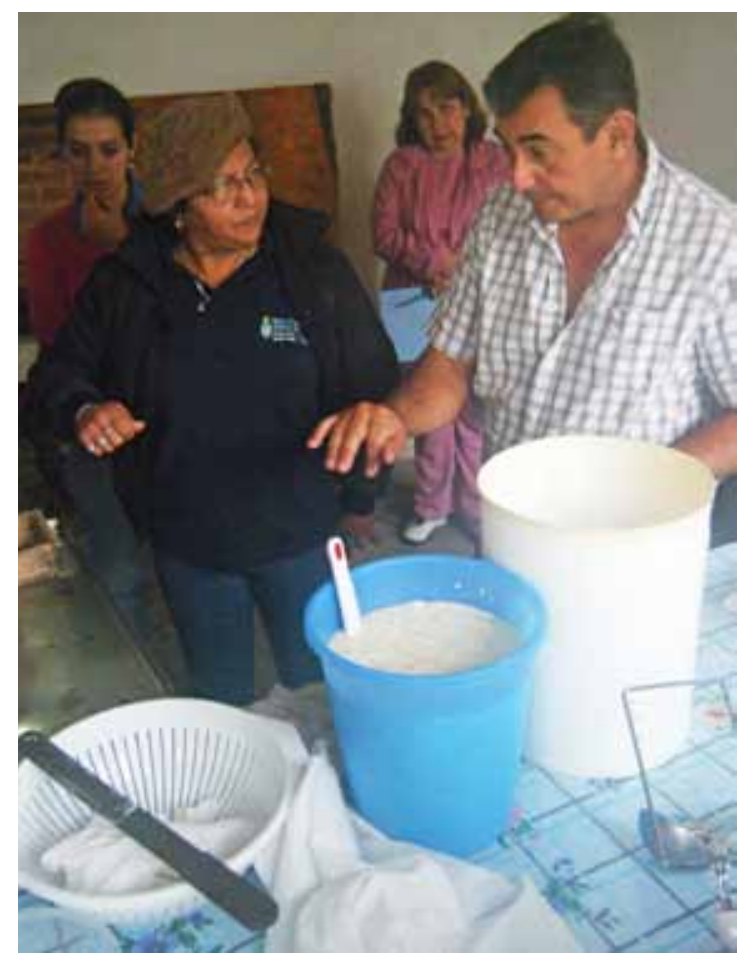

Figura1.Productores cortandolacuajadaengranos.
Prácticas de manufactura en cada etapa del proceso.En cada una de ellas, la participación individual de cada uno de los productores, enriqueció la actividad con sus experiencias particulares, basadas en la tradición (Fig.1).

La preparación del agente coagulante artesanal para su uso fue desarrollada en particular, a fin de transmitir los resultados experimentales del Grupo BIMIA al respecto. Destacando que es posible emplear este agente coagulante autóctono que contribuye a las características típicas de este queso regional, no adiciona contaminantes biológicos y no representa riesgo de transmisión de Encefalopatía Espongiforme Bovina (Vasek y Falcione, 2013b).

La elaboración del agente coagulante se lleva a cabo en dos etapas. La primera consiste en la conservación del material primario: cuajar de bovino adulto, lavado, salado y secado en condiciones experimentales determinadas, que proveen un insumo de duración prolongada (3-5 años) si se lo conserva en ambiente seco y frío. A tal fin, en nuestro laboratorio, se diseñó un secadero de fácil construcción y bajo costo, de modo tal que los mismos productores de quesos puedan fabricarlo en madera estacionada impermeabilizada con sistema de calefacción generado por bombillas de luz y homogeneización del aire caliente mediante provisión de dos circuladores de aire internos. En la segunda etapa, se sumerge un trozo de cuajar seco-salado en leche (relación $60 \mathrm{~g} / \mathrm{L}$ ) y el suero exudado más los trozos de tejido se trasvasan a un frasco limpio y estéril para su empleo (Fig. 2).

En forma complementaria, se encuestó a los productores a fin de elaborar un diagnóstico en referencia a sus características productivas: número de personas 


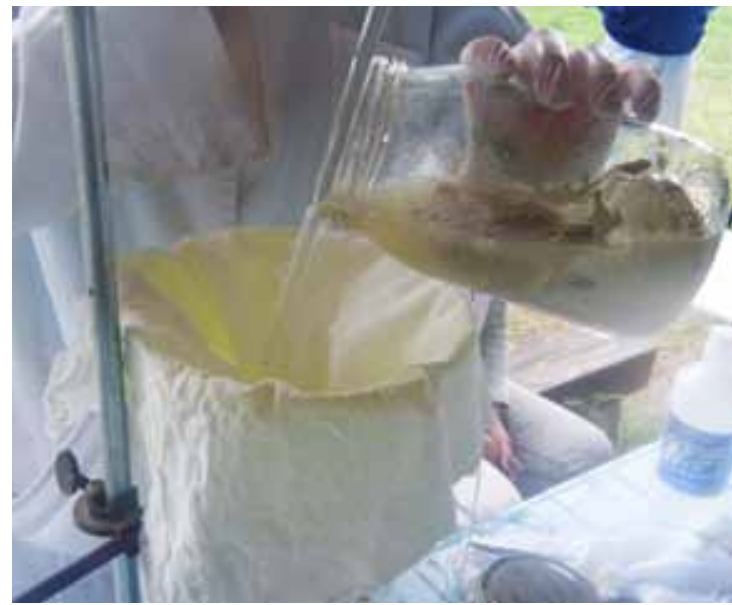

Figura 2. Etapa final de la preparación de agente coagulante artesanal.

involucradas, disponibilidad de ingreso monetario adicional, nivel de manufactura diaria y metas productivas a futuro.

\section{RESULTADOS Y DISCUSIÓN}

Durante la Jornada se corroboró la experticia que van adquiriendo los productores, en particular, el responsable del Establecimiento donde se realizó esta experiencia capitalizó la importancia del empleo del acero inoxidable como material de construcción para los equipos, dado que luego del Taller-2014, construyó en ese material los moldes para sus quesos y una mesa desueradora (Fig. 3).

Los resultados de la encuesta realizada señalaron que todos los productores asistentes, emplean leche de su propio ganado vacuno, agente coagulante preparado artesanalmente, sin la adición de fermento comercial. No consideran como competencia a otros productores y aspiran a mejorar sus prácticas y aumentar el volumen de producción. Estos resultados, entre otros (Tabla 1, preguntas 1-4), muestran un claro interés por mantener la tradición, continuar con la elaboración y no desvirtuar el carácter artesanal de sus productos.

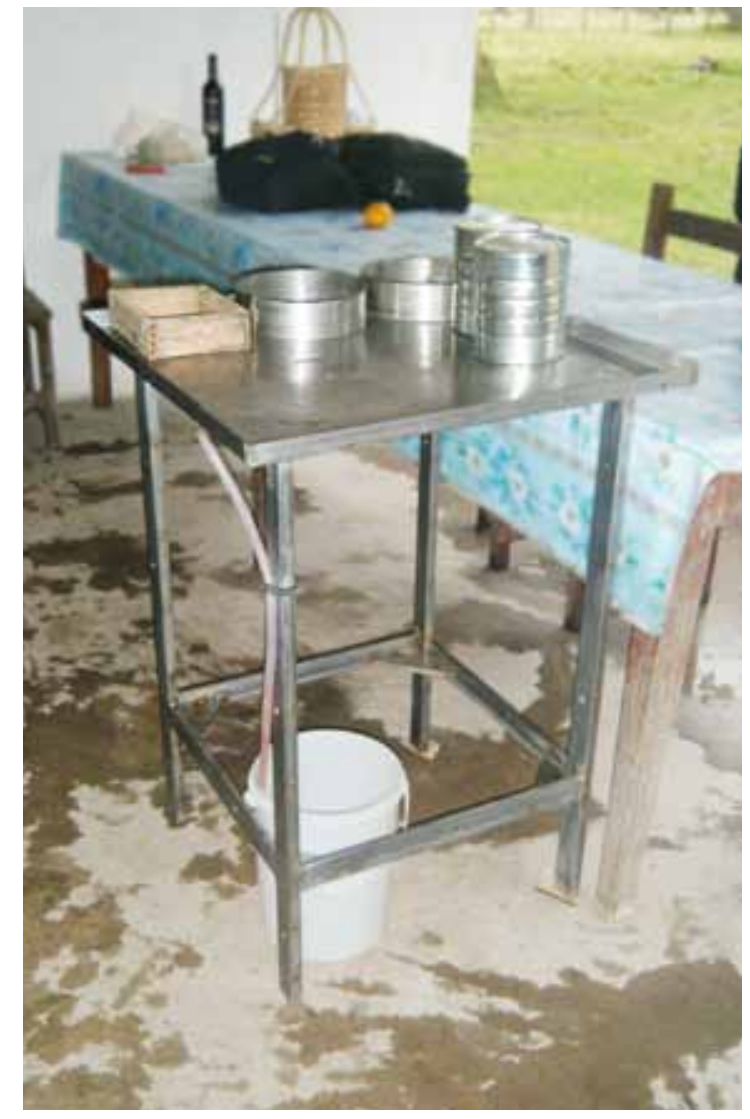

Figura 3. Utensilios construidos en acero inoxidable por un productor, junto a un molde de madera empleado antiguamente.

Un pequeño porcentaje de este grupo de productores $(15,38 \%)$ genera, además, trabajo a otras familias y posee un nivel de producción quesera no despreciable. Sin embargo, la mayor venta de los quesos, continua efectuándose entre vecinos y en Ferias Francas. Desde el punto de vista de una de las misiones de la Universidad Nacional del Nordeste, y del Grupo BiMIA en particular, respecto a la devolución a la sociedad de los conocimientos generados en sus claustros, queda claro el respaldo e impulso que es necesario efectuar a este sector productivo, en pos del crecimiento regional y desarrollo sustentable, en un todo de acuerdo con el Programa Nacional Argentina 2020. 


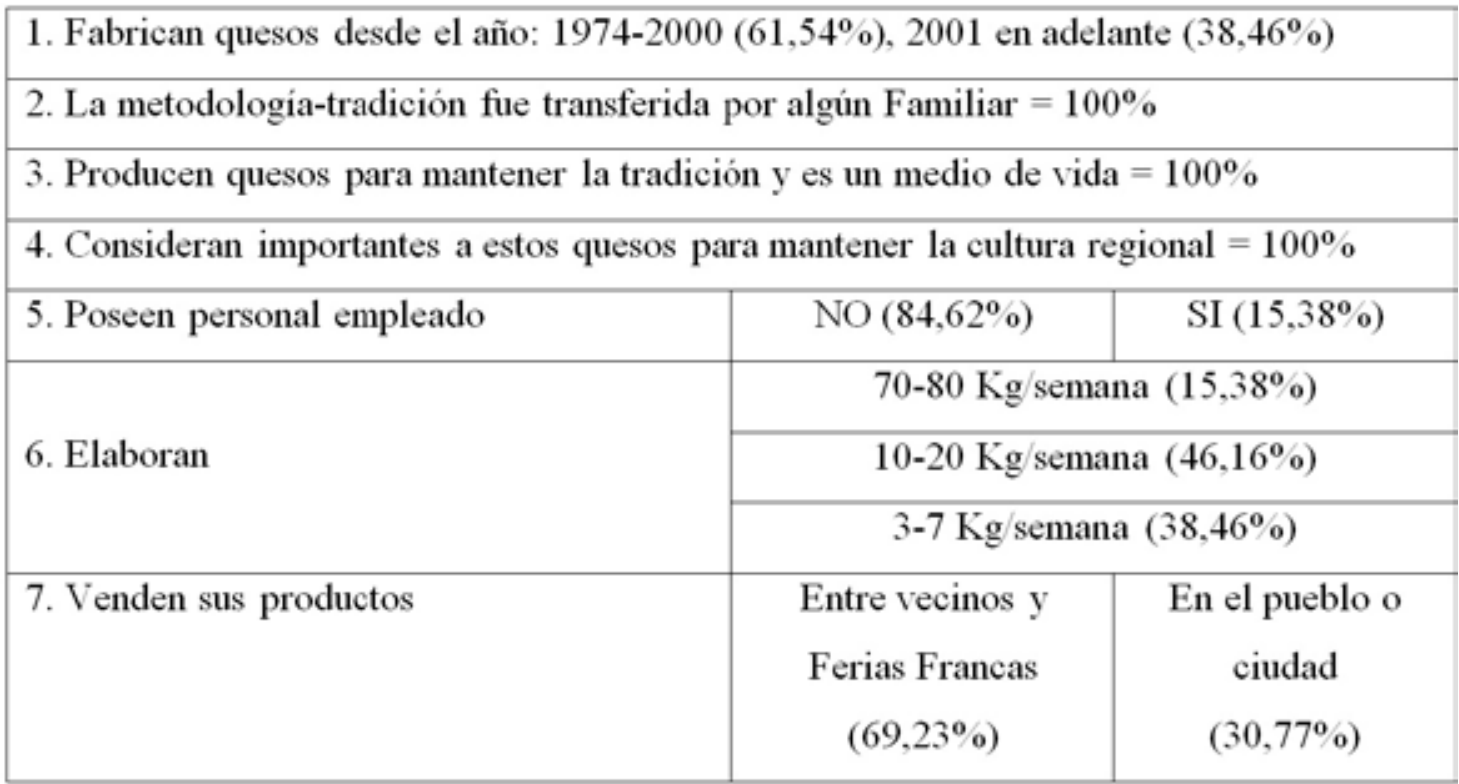

Tabla 1. Estadísticos porcentuales resultantes de la encuesta a los productores.

\section{Agradecimientos}

El Grupo BiMIA agradece especialmente la colaboración en la gestión logística al Sr. José Valenzuela de la localidad de San Luis del Palmar.

Referencias

Vasek OM, LF Falcione. 2013a. Strategy cocreatin value for cheese production in Ramada Paso-Itati, Corrientes, Argentina, exploiting the local food resource. Proceeding VI Congresso Internacional Sistemas Agroalimentarios Localizados. ISBN: 978-287614-691-4. 2:1-29. Florianópolis, Brasil.
Vasek OM, LF Falcione. 2013b Quesos Artesanales de Corrientes. Riesgo de Enfermedades Transmitidas por alimentos: encefalopatía espongiforme bovina. 2013. FaCENA, ISN 0325-4216, 27:13-23.

Vasek OM, JG Leblanc, A Fusco, GS de Giori. 2008. Chemical composition and microbial evaluation of Argentinean Corrientes 